Widefield ScIENCE AND TeChNOLOGY For the SKA

SKADS CONFERENCE 2009

S.A. Torchinsky, A. van Ardenne, T. van den Brink-Havinga, A.J.J. van Es, A.J. Faulkner (eds.)

4-6 November 2009, Château de Limelette, Belgium

\title{
Radio Transients and Aperture Arrays
}

\author{
T.J.W. Lazio ${ }^{1,2}$ \\ 1 Naval Research Laboratory, 4555 Overlook Ave. SW, Washington, DC 20375-5351 USA \\ 2 SKA Program Development Office
}

\begin{abstract}
A series of discoveries over the past decade have illustrated that the radio sky may be quite dynamic—known sources have been discovered to behave in new ways and what may be entirely new classes of sources have been discovered. I provide an incomplete list of the variety of transients that might be accessible to the SKA and consider aspects of the SKA design relevant to the detection and characterization of various kinds of transients. Aperture arrays have had an important role in previous discoveries of radio transients, most notably in the discovery of pulsars, and aperture arrays could prove useful for transient studies with the SKA.
\end{abstract}

\section{Introduction}

Observations at radio wavelengths have been responsible for much of what we regard today as modern astronomy: the entire concept of non-thermal emission resulted from early radio observations of the Milky Way Galaxy while the discovery or recognition of the Cosmic Microwave Background, active galactic nuclei, pulsars, masers, and extrasolar planets all happened at radio wavelengths. Moreover, because the Universe is so faint, many observations, at wavelengths across the spectrum, assume that the sky is unchanging so as to obtain long integration times and improved sensitivity. Indeed, the technique of aperture synthesis - the development of which Sir Martin Ryle was awarded the 1974 Nobel Prize in Physics for his work in radio aperture synthesis-implicitly assumes that the object(s) being observed is unchanging.

Much of astronomy's progress over the last half of the $20^{\text {th }}$ Century resulted from opening new spectral windows, including at radio wavelengths. With essentially the entire electromagnetic spectrum having been explored at some level, we must look to other regions of parameter space-such as increased sensitivity, field of view, or the time domain-for future transformational discoveries. The time domain appears ripe for new exploration as observations over the past decade have emphasized that the sky may be quite dynamic - known sources have been discovered to behave in new ways and what may be entirely new classes of sources have been discovered.

Beyond a simple appeal to expanding parameter space, and even if discoveries of new classes of radio transients are not in any way transformational, the study of radio transients is well motivated for the following reasons: Transient sources are necessarily compact, so that they mark the location of explosive or dynamic events and can be used to probe fundamental physics and astrophysics. Further, transient signals carry information about the medium(a) through which they have propagated.

Here, I provide several examples to motivate the range of transients that the SKA or its Precursors and Pathfinders might be able to study as well as some thoughts about design considerations for the SKA in order to enable it to conduct transient observations.

\section{The Radio Transient Sky}

We begin with a brief, and incomplete, sampling of the kinds of radio transients that are either known or hypothesized with an eye toward motivating both expanded work as well as emphasizing the broad range of physics that radio transients can probe.

Planets-Solar and Extrasolar Jupiter has long been known to produce decameter bursts (Burke \& Franklin 1955), and all of the planets with strong magnetic fields (Earth, Jupiter, Saturn, Uranus, and Neptune) produce radio radiation via the electron cyclotron maser instability, though only Jupiter's emission is accessible from the ground. Even for the well-studied case of Jupiter, fundamental questions remain about the location and origin of the its radio emission. Jupiter's decametric emission occurs below $40 \mathrm{MHz}$, which is below the nominal lower frequency limit of the SKA. However, this will be accessible to the International LOFAR Telescope, and preliminary observations already appear promising (Nigl et al. 2007).

At least one of the known extrasolar planets also appears to have a strong magnetic field (Shkolnik et al. 2005). By analogy to the solar system planets, there have been a host of suggestions (e.g., Yantis et al. 1977; Winglee et al. 1986; Zarka et al. 1997; Farrell et al. 1999)_-some predating the discovery of extrasolar planets - that extrasolar giant planets also produce bursty emission, presumably at frequencies below $100 \mathrm{MHz}$. Detection of radio emission from extrasolar planets would constitute direct detection, in contrast to the largely indirect detections of the known extrasolar planets obtained from the reflex motions of their host stars. Moreover, as the magnetic fields of the solar system planets are thought to be generated deep within their interiors, the radio emission from extrasolar planets could provide an indirect probe of the interiors of extrasolar planets.

Brown Dwarfs Radio flares have been detected from a number of brown dwarfs (Berger et al. 2001; Berger 2002; Hallinan et al. 2007). The flares are thought to originate in magnetic activity on the surfaces of the brown dwarfs, 
but many objects have radio emission that deviates from the expected radio-X-ray correlation observed for stars (Berger 2006). In some notable cases, the radio emission is pulsed, consistent with a "lighthouse"-like effect in which the emission arises from the magnetic pole of a rotating brown dwarf, not dissimilar to pulses from radio pulsars. In contrast to pulsar emission, however, the radio emission of brown dwarfs has been identified as being due to the electron cyclotron maser instability (Hallinan et al. 2008). The majority of brown dwarf observations have been conducted at frequencies above $5 \mathrm{GHz}$, consistent with relatively strong magnetic fields, while lower frequency emission is only poorly constrained.

Flare Stars Radio flares from dwarf stars have been known for some time (Hjellming 1988), and many low-mass stars (spectral types K and M) show significant "radio activity," often with radio flares or bursts on short time scales (Osten \& Bastian 2008). This radio emission is thought to be linked to coronal processes on the stars, likely closely coupled to the magnetic field structure. Study of the coronal processes in these extreme cases may provide understanding of solar processes, which could impact not only astrophysics but aspects of the Earth-Sun connection as well (e.g., space weather, climate).

Neutron Stars The discovery of radio pulses from neutron stars (Hewish et al. 1968) provided the first confirmation that such extreme objects were not simply theoretical artifices (Baade \& Zwicky 1934). Since that time, observations of radio pulsars have been spectacularly successful in probing various aspects of fundamental physical theories and observations of radio pulsars have resulted in the 1974 and 1993 Nobel Prizes in Physics. In addition to the "standard" radio pulsar, however, recent discoveries emphasize that the transient radio emission from neutron stars may be much broader than had been previously appreciated. Two examples are "transient" pulsars and rotating radio transients (RRATS).

Transient pulsars are a class of pulsars for which the pulsed emission itself shows intervals of being "on" and "off." For the particular case of PSR B1931+24, the pulsar exhibits a secular decrease in its period, similar to that seen for other pulsars and consistent with the loss of rotational energy. However, during the intervals when it is producing radio pulses (when it is "on"), it shows an elevated energy loss rate, as predicted for a rotating magnetic dipole, and providing insight into the plasma physics in the pulsar magnetosphere (Kramer et al. 2006).

RRATs are a class ${ }^{\mathrm{a}}$ of neutron stars that is distinguished by the fact that they are detectable only via single radio pulses (McLaughlin et al. 2006). At a single stroke, the discovery of RRATs essentially led to a doubling of the estimated neutron star population of the Galaxy, which has led to the

\footnotetext{
a The discovery of RRATs shares certain similarities with the discovery of pulsars. In both cases, a young astronomer examined a significant amount of data from a radio telescope, and she was able to notice occasional signals that would ultimately be identified as a new class of objects.
}

(recent) suggestion that the Galactic supernova occurrence and neutron star birth rates are not in balance (Keane \& Kramer 2008). RRATs have also been detected in the Xray (McLaughlin et al. 2007), which has helped both to elucidate, and to put into sharp relief, the range of properties shown by neutron stars: RRATs show some similiarities to extremely strong magnetic field neutron stars (magnetars), but other similarities to "standard" radio pulsars.

More generally, pulsars have long been known to undergo "nulling" events (Backer 1970). These nulls may also provide information about the plasma magnetosphere, or even indicate the presence of sub-stellar companions (i.e., asteroids) to pulsars (Li 2006; Cordes \& Shannon 2008).

Pulsar Giant Pulses While pulsar giant pulses are a subset of the radio phenomenology exhibited by radio pulsars, I discuss them separately here in order to highlight the yin- $\int$ yang nature of transient studies. Some pulsars emit "giant" pulses, with strengths that are 1000 times or more the mean pulse intensity. In the case of the Crab pulsar, some giant pulses outshine the entire Crab Nebula, on time scales of roughly $100 \mu$ s (Hankins \& Rickett 1975), corresponding to a brightness temperature of $10^{31} \mathrm{~K}$. Among the most extreme pulses ever detected are a series of nanosecond duration pulses, recorded using specially-built hardware at frequencies above $5 \mathrm{GHz}$ (Hankins et al. 2003); their im- $\Omega$ plied brightness temperatures exceed $10^{38} \mathrm{~K}$, making them the most luminous emission from any astronomical object. For many years, this phenomenon was thought to be unique to the Crab pulsar, but giant pulses have since been detected from other pulsars (Cognard et al. 1996; Johnston \& Romani 2003). With their short durations, giant pulses must originate from regions perhaps only meters in size within the pulsar magnetosphere, providing an exquisite probe of extreme plasma magnetohydrodynamics. Concurrently, because of their brightness, giant pulses could potentially be detected from exceedingly distant pulsars, including those in other galaxies. Current models for large-scale structure formation predict that matter in the current epoch forms a "cosmic web" (Cen \& Ostriker 1999; Davé et al. 1999, 2001). Most of the baryons in the Universe reside in large-scale filaments that form the "strands" of the web, with groups and clusters of galaxies located at the intersections of filaments. At the current epoch, hydrogen gas continues to accrete onto and stream along these filaments, undergoing multiple shocks as it falls into the gravitational potential wells located at the intersections of the filaments. The filaments of hydrogen form a warm-hot ionized medium (WHIM), with a temperature of $10^{5}-10^{7} \mathrm{~K}$. Observations of highly ionized species of oxygen and neon by both the FUSE and Chandra observatories are considered to be validations of these predictions. Absorption observations along various lines of sight suggest a diffuse medium with a temperature of order $10^{6} \mathrm{~K}$ with a density of order $5 \times 10^{-5} \mathrm{~cm}^{-3}$ (e.g., Savage et al. 2005; Howk et al. 2009). While striking, these observations still suffer from the difficulty of probing only trace elements. The ionized hydrogen in the WHIM has not been detected directly. Radio pulses exhibit dispersion due to the 
frequency-dependent nature of the (interstellar) plasma's refractive index, with the amount of dispersion depending directly upon the ionized hydrogen column density. Pulses from extragalactic pulsars should exhibit additional dispersion due to the plasma in the Local Group medium or intergalactic medium, depending upon their distances. Thus, pulsar giant pulses have the potential to probe directly the most abundant element in the Local Group medium or WHIM. Current searches have yielded intriguing, but not yet confirmed, candidates for extragalactic pulsars in M33 McLaughlin \& Cordes (2003) from a search at the Arecibo Observatory, and the SKA should be able to search much deeper in the Local Supercluster.

Unknown Transients As an example of the potential discoveries awaiting transient searches, I consider two largely archival searches for transients. The VLA and GMRT have been used to monitor the Galactic center at $330 \mathrm{MHz}$, a frequency at which both telescopes naturally provide wide fields of view. A total of three transients have been discovered, (Hyman et al. 2002, 2005, 2009; Spreeuw et al. 2009), the most notable of which is GCRT J1745-3009. This source has been detected both at the VLA and GMRT, with the detection at the VLA revealing several bursts (duration $\sim 10 \mathrm{~min}$.) during a 6-hr observation. A number of possible explanations has been advanced, from a white dwarf pulsar to a double neutron star system to a precessing radio pulsar to a flaring brown dwarf (Zhang \& Gil 2005; Turolla et al. 2005; Zhu \& Xu 2006; Hallinan et al. 2007), but our understanding of this source remains quite limited, with only limited spectral information and few constraints on distance. The few constraints arise in part because the searches are largely archival in nature, so by definition there is no ability to obtain contemporaneous measurements, but they also illustrate the need for both a large frequency range and frequency agility.

Lunar Neutrino Bursts Ultra-high energy cosmic rays (UHECRs), with energies exceeding $10^{18} \mathrm{eV}$, have been detected with numerous particle detectors. These particles have energies well beyond what can be achieved in terrestrial accelerators and must originate from some of the most extreme environments in the Universe. However, because of the Greisen-Zatsepin-Kuzmin (GZK)-limit (Greisen 1966; Zatsepin \& Kuzmin 1966), particle propagation is limited to a horizon of order $50 \mathrm{Mpc}$. The same accelerators that produce UHECRs might also be expected naturally to produce ultra-high energy neutrinos. Thus, ultra-high energy neutrinos offer a possible means of probing beyond our particle horizon. Particles beyond those in the Standard Model in particle physics, potentially including dark matter particles, also could produce ultra-high energy neutrinos as a decay product. Generically, models that extend the Standard Model or grand unified theories (GUTs) predict unstable particles (e.g., Berezinsky 2006). Recently, there was intense interest in such models, so-called "top down" sources of ultra-high energy neutrinos, and the resulting diffuse flux of ultra-high energy particles, as the result of initial reports of a violation of the GZK limit. Ultra-high energy neutrinos impacting the lunar regolith should produce radio pulses near $1 \mathrm{GHz}$ (Dagkesamanskii \& Zheleznykh 1989; Saltzberg et al. 2001), though searches to date have been unsuccessful (Hankins et al. 1996; Gorham et al. 2004; Beresnyak et al. 2005; Scholten et al. 2006, 2009; Jaeger et al. 2009).

Ultra-high energy neutrinos are of particular interest as their propagation is essentially unimpeded and can be used to probe the highest energies to which cosmic sources can accelerate particles as well as potentially to indicate physics beyond the Standard Model (e.g., Halzen \& Hooper 2002). Moreover, given the low flux levels expected for the highest energy neutrinos, the Moon presents an attractive target as essentially its entire limb acts as the "detector," with a volume substantially larger than is feasible for terrestrial detectors (volumes $\sim 1 \mathrm{~km}^{2}$, e.g., IceCUBE).

Evaporating Black Holes Rees (1977) first suggested that $\Omega$ the evaporation of primordial black holes would generate a radio pulse, and O'Sullivan et al. (1978) and Phinney \& Taylor (1979) have conducted searches for such ra-

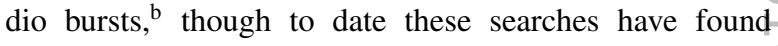
no convincing signals. More recently, advances in $\gamma$-ray detectors, particularly at $\mathrm{TeV}$ energies, has renewed interest in possible high-energy signatures from primordial black holes (Dingus et al. 2002; Linton et al. 2006). Observations at the extremes of the electromagnetic spec- $\int$ trum are complementary as radio observations attempt to detect the pulse from an individual primordial black hole, while high-energy observations generally search for the integrated emission from a population (e.g., in the Galaxy or Local Group).

Gravitational Wave Events The progenitors for various gravitational wave events may generate associated electromagnetic signals or pulses. A notable example is the inspiral of a binary neutron star system, which is one of the key targets for LIGO (Abbott et al. 2006). As a binary neutron star inspirals, the interaction of the magnetospheres of the neutron stars can produce electromagnetic pulses, probably at both high energies and at radio (e.g., Hansen \& Lyutikov 2001). More generally, the combined detection of both electomagnetic (including radio) and gravitational wave signals may be required to produce useful localizations and understanding of the gravitational wave emitters (Kocsis et al. 2007; Bloom et al. 2009).

Extraterrestrial Civilizations While no such examples are known of this class, many searches for extraterrestrial intelligence (SETI) find non-repeating signals that are otherwise consistent with the expected signal from an extraterrestrial transmitter. Cordes et al. (1997) discuss how extraterrestrial transmitters could appear to be transient, even if intrinsically steady. Further, the detection of ET transmitters might occur not as a result of a directed SETI program, but serendipitously from a general search of the sky. As Tarter (2007, private communication) has pointed out, the

\footnotetext{
b One of these searches led to J. O'Sullivan developing the techniques for the 802.11 wireless communication protocol, for which he was recently awarded the Prime Minister's Prize for Science in Australia.
} 
behavior of PSR B1931+24 is strikingly similar to what might be expected if a civilization was going to send a directed signal. That is, one way that a civilization might increase the probability of its signals being detected is to make them appear similar to, but strikingly different, than a class of signals for which other civilizations might be searching. For instance, the increase in the energy loss rate from PSR B1931+24 during the intervals when it is "on" is consistent with that expected from pulsar emission models, but if the energy loss rate was observed to decrease while during its "on" phase, an alternate explanation for such a "pulsar" would have to be considered. ${ }^{\mathrm{c}}$

\section{Radio Transients and the Design of the SKA}

I begin with a specific example of transient hunting with a sparse aperture array. Figure 1 shows the sky at $61 \mathrm{MHz}$, as observed by the Long Wavelength Demonstrator Array (LWDA). This 16-element dipole phased array was constructed by the Applied Research Laboratory of the University of Texas at Austin, under contract from the Naval Research Laboratory. The LWDA operational frequency range was 60 to $80 \mathrm{MHz}$, and its objective was to serve as a technical testbed for a future Long Wavelength Array station. During the course of its operation, a simple imaging correlator was developed, and, as the field of view of the individual dipoles is close to $2 \pi$ sr, the LWDA could image the full sky, albeit in a narrow bandwidth mode.

\footnotetext{
${ }^{c}$ It is worth recalling that the first few pulsars discovered were named LGM, for "little green men," as Bell, Hewish, and their collaborators considered it initially plausible that the periodic signals that they had detected might have been generated intelligently.
}

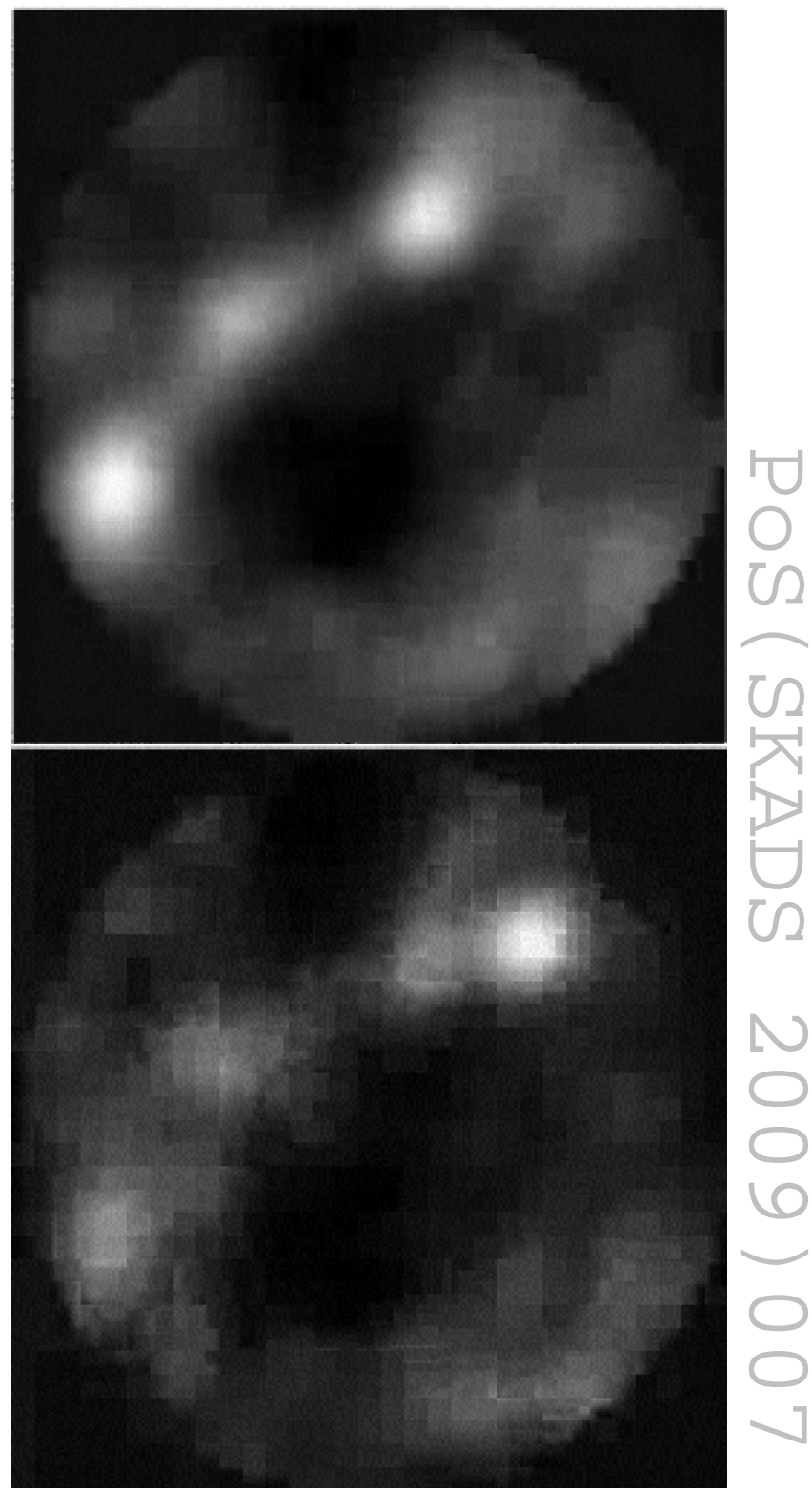

Fig. 1: Illustration of the potential of aperture arrays for radio transient detection. The Long Wavelength Demonstrator Array (LWDA) was a 16-element dipole phased array located on the VLA site in New Mexico; in SKA parlance, it was a "sparse aperture array." Both images shown here are at $61 \mathrm{MHz}$ and were acquired in 2006 November, during the Leonid meteor shower. (Top) An all-sky LWDA image, with an integration time of $0.1 \mathrm{~s}$. The Galactic plane slopes diagonally from the upper right to the lower left and the sources Cyg A and Cas A are visible in addition to a general enhancement toward the inner Galaxy. (Bottom) An image acquired only seconds later. The dominant source is a TV transmitter located hundreds of kilometers away, which is reflecting off an ionized meteor trail. 
It has long been known that as a meteor enters the Earth's atmosphere, its velocity is high enough that it can produce a ionized trail, which can reflect a transmitted radio signal (e.g., Millman et al. 1948). The LWDA was operated during the Leonid meteor shower in 2006 (November). At the time that these images were formed, various television stations in New Mexico were operating on a frequency near $61 \mathrm{MHz}$. In the course of the observational run during the Leonid meteor shower, many "transients" were seen, consistent with TV transmitter reflections off ionized meteor trails.

While these transients can be considered astronomical only in the most generous of definitions, the detection and imaging of transients with the LWDA does illustrate that an aperture array with a wide field of view does have potential as "transient hunting machine." I now turn to the more general question of radio transient hunting with the SKA.

In the Rayleigh-Jeans limit, the brightness temperature $T_{B}$ of a transient is

$$
T_{B} \propto \frac{S D^{2}}{(W v)^{2}},
$$

where the combination of the source's flux density $S$ and distance $D$ is equivalent to a "pseudo-luminosity" and the combination of the observational frequency $v$ and transient (pulse) width $W$ are an "uncertainty-like" quantity. This description of the brightness temperature leads naturally to a transient "phase space." Figure 2 shows how both known and hypothesized classes of radio transients populate this phase space. Notable about this phase space is both its size-just the known populations of transients cover nearly 20 orders of magnitude in pseudo-luminosity $S D^{2}$ and 15 orders of magnitude in $W v-$ and the extent to which Nature has produced different kinds of sources to populate this phase space. With respect to the SKA, one could design a series of transient search experiments in which the observational frequency $v$, cadence, time resolution, and limiting flux density were used to probe different regions of the phase space, with a particular eye to finding new classes of transients that would populate the phase space more fully.

An alternate approach is to characterize the design goals of the SKA in terms of previous observations or observations that could be carried out by Pathfinders or Precursors. The advantage of this approach is that it is likely to preserve the overall flexibility of the instrument and is likely to increase the likelihood of finding unknown classes of sources. Figure 3 considers telescope gain ${ }^{\mathrm{d}} G\left(\mathrm{~K} \mathrm{Jy}^{-1}\right)$ and the (FWHM) of the instantaneously accessible field of view $\theta$ (arcminutes) as functions of frequency. This parameter space approach can also be related to a transient source survey speed figure of merit (Cordes 2007),

$$
\mathrm{FoM}_{t}=\Omega\left(\frac{A_{\text {eff }}}{T_{\text {sys }}}\right)^{2} K(\eta W, \tau W),
$$

which is a function of the telescope sensitivity $A_{\text {eff }} / T_{\text {sys }}$, instantaneous solid angle $\Omega$, typical time duration of the transient $W$, event rate $\eta$, and the time per telescope pointing

\footnotetext{
$\mathrm{d}$ The gain can be related to the more commonly used measure of sensitivity within the SKA community as $G T_{\text {sys }}=2 k_{B}\left(A_{\text {eff }} / T_{\text {sys }}\right)^{-1}$ Thus, $1 \mathrm{~K} \mathrm{Jy}^{-1}$ implies a sensitivity of $55 \mathrm{~m}^{2} \mathrm{~K}^{-1}$ and $10000 \mathrm{~m}^{2} \mathrm{~K}^{-1}$ implies a gain of $5.5 \times 10^{-3} \mathrm{~K} \mathrm{Jy}^{-1}$, with both conversions using an assumed $T_{\text {sys }}=50 \mathrm{~K}$.
}

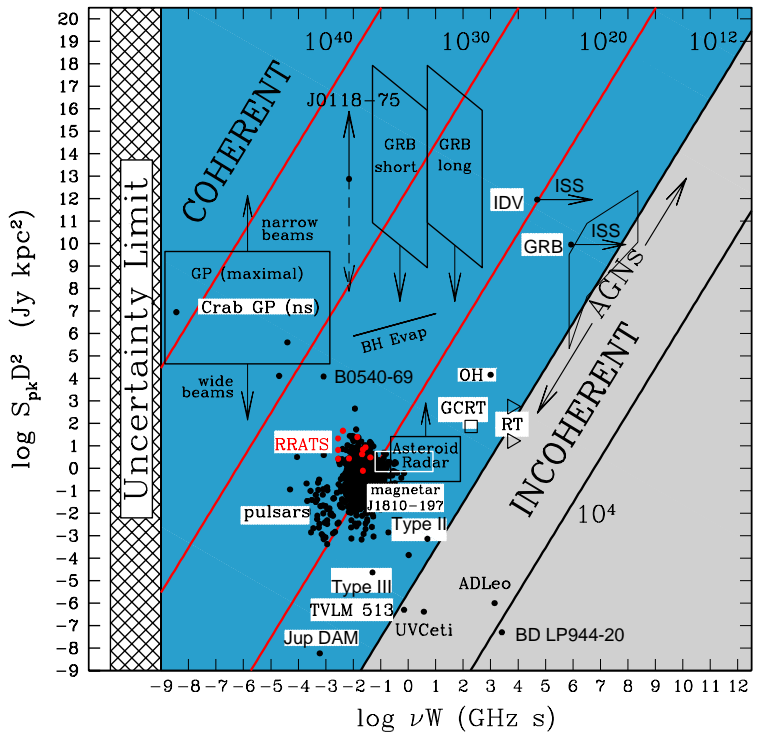

Fig. 2: Phase space for radio transients. The abscissa is the prod uct of the observation frequency $v$ and transient duration or pulse width $W$; the ordinate is the product of the flux density $S$ and square of the distance $D^{2}$. In the Rayleigh-Jeans approximation, these quantities are directly proportional and related to the brightness temperature $T_{B}$ (eqn. 1). The sloping lines are labelled by constant brightness temperature, with $T_{B}=10^{12} \mathrm{~K}$ taken to divide coherent from incoherent sources. Examples of transient emission from various classes of sources are indicated. In the case of gamma-ray burst afterglows (GRB) and intraday variability (IDV) of active galactic nuclei (AGN), the apparently high brightness temperatures are not thought to be intrinsic but related to interstellar scintillation (ISS). For these two classes of sources, how the absence of ISS would affect their locations in this phase space is also shown. In addition to known examples of transients, the locations of hypothesized radio transients also are shown, including black hole evaporation ("BH Evap") and prompt radio emission from GRBs ("GRB short" and "GRB long"). (Figure courtesy of J. Cordes.)

("dwell time") $\tau$. The function $K(\eta W, \tau W)$ incorporates the likelihood of detecting a particular kind of transient.

Roughly, one can separate transients surveys into two classes: (1) Burst searches that probe timescales of less than about $1 \mathrm{~s}$ for which large $\Omega$, perhaps at the expense of $A_{\text {eff }} / T_{\text {sys }}$, are most appropriate; and (2) Imaging surveys conducted with interferometers that typically probe timescales of tens of seconds and longer and for which large values of $A_{\text {eff }} / T_{\text {sys }}$, but relatively small values of $\Omega$, have been used. Both a challenge and opportunity for development work related to the SKA- and a potential for aperture arrays - is the extent to which a "large" $\Omega$ and $A_{\text {eff }} / T_{\text {sys }}$ could be obtained simultaneously.

\section{Acknowledgements}

I thank G. Bower, J. Cordes, and P. Dewdney for helpful discussions. Basic research in radio astronomy at the NRL is supported by 6.1 Base funding. 

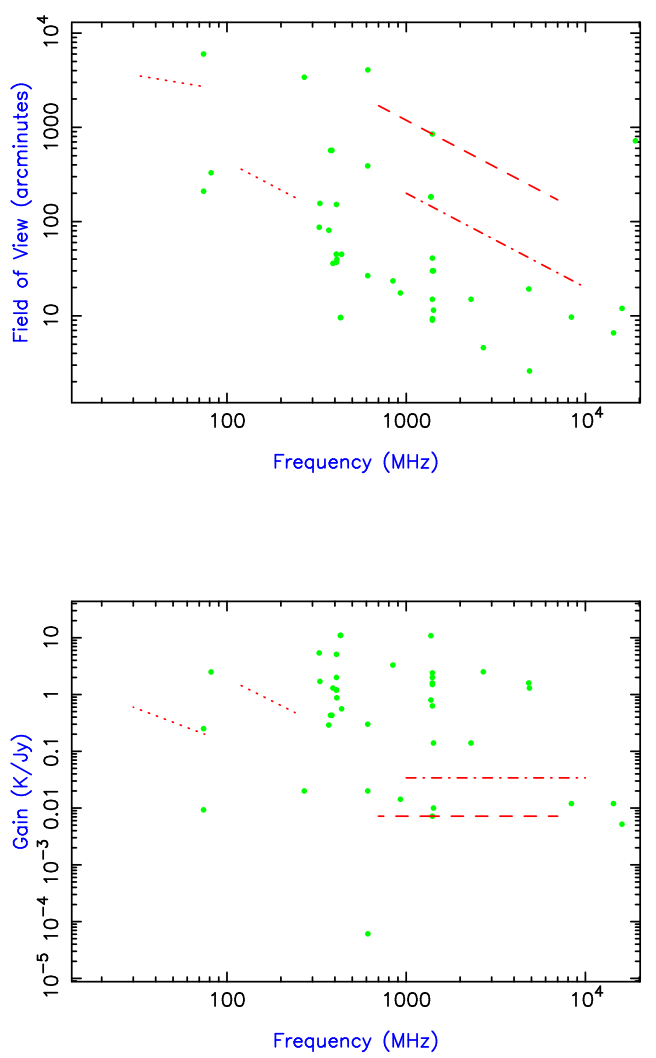

Fig. 3: Parameter space relevant for SKA transient hunting. (Top) The (FWHM) instantaneously accessible field of view as a function of frequency. For reference, a field of view of $100^{\prime}$ is equivalent to $2.8 \mathrm{deg}{ }^{2}$ (Bottom) The telescope gain as a function of frequency. In both panels, the points indicate the field of view or gain of a telescope on which a transient hunting program has been conducted. The sloping lines show the fields of view accessible to the ATA (dashed line), EVLA (dot-dashed line), and LOFAR (dotted line), were they to be operated in a "fly's eye" mode (Bower et al. 2009); for the ATA, an effective frequency range of $0.7-7 \mathrm{GHz}$ is adopted, as the sensitivity of the telescope is decreasing outside this range, and, for the EVLA, an upper frequency limit of $10 \mathrm{GHz}$, consistent with the nominal value for SKA-mid, is adopted. Shown is one of the many operational modes for LOFAR. The data shown may not be complete, but can be viewed as illustrative with respect to the SKA.

\section{References}

Abbott, B., Abbott, R., Adhikari, R., et al. 2006, Phys. Rev. D, 73, 102002

Baade, W., \& Zwicky, F. 1934, Phys. Rev., 46, 76

Backer, D. C. 1970, Nature, 228, 42

Beresnyak, A. R., Dagkesamanskii, R. D., Zheleznykh, I. M., Kovalenko, A. V., \& Oreshko, V. V. 2005, Astron. Rep., 49, 127

Berezinsky, V. 2006, Nuclear Phys. B Suppl., 151, 260

Berger, E. 2006, ApJ, 648, 629

Berger, E. 2002, ApJ, 572, 503

Berger, E., Ball, S., Becker, K. M., et al. 2001, Nature, 410, 338
Bloom, J. S., Holz, D. E., Hughes, S. A. 2009, arXiv:0902.1527

Burke, B. F. \& Franklin, K. L. 1955 JGR, 60, 213-217

Bower, G. C., et al. 2009, in preparation

Cen, R. \& Ostriker, J. P. 1999, ApJ, 514, 1

Cognard, I., Shrauner, J. A., Taylor, J. H., \& Thorsett, S. E. 1996, ApJ, 457, L81

Cordes, J. M. 2007, SKA Memorandum \#97, http://www. skatelescope.org

Cordes, J. M., \& Shannon, R. M. 2008, ApJ, 682, 1152

Cordes, J. M., Lazio, T. J. W., \& Sagan, C. 1997, ApJ, 487, 782

Dagkesamanskii, R. D., \& Zheleznykh, I. M. 1989, Sov. Phys. JETP Lett., 50233

Davé, R., Cen, R., Ostriker, J. P., et al. 2001, ApJ, 552, 473

Davé, R., Hernquist, L., Katz, N., \& Weinberg, D. H. 1999 ApJ, 511, 521

Dingus, B., Laird, R., \& Sinnis, G. 2002, 34 $4^{\text {th }}$ COSPAR 2 Scientific Assembly, \#2744

Farrell, W. M., Desch, M. D., \& Zarka, P. 1999, JGR, 104, 14025

Gorham, P. W., Hebert, C. L., Liewer, K. M., Naudet, C. J., Saltzberg, D., \& Williams, D. 2004, Phys. Rev. Lett., 93 041101

Greisen, K. 1966, Phys. Rev. Lett., 16, 748

Hallinan, G., Antonova, A., Doyle, J. G., Bourke, S., Lane, C. \& Golden, A. 2008, ApJ, 684, 644

Hallinan, G., Bourke, S., Lane, C., et al. 2007, ApJ, 663, L25

Halzen, F., \& Hooper, D. 2002, Rept. Prog. Phys., 65, 1025

Hankins, T. H., Kern, J. S., Weatherall, J. C., \& Eilek, J. A. 2003, Nature, 422, 141

Hankins, T. H., Ekers, R. D., \& O'Sullivan, J. D. 1996, MNRAS, 283, 1027

Hankins, T. H. \& Rickett, B. J. 1975, Methods in Computational Physics. Volume 14 - Radio Astronomy, 14, 55

Hansen, B. M. S., \& Lyutikov, M. 2001, MNRAS, 322, 695

Hewish, A., Bell, S. J., Pilkington, J. D. H., Scott, P. F., \& Collins, R. A. 1968, Nature, 217, 709

Hjellming, R. M. 1988, in Galactic and Extragalactic Radio Astronomy, eds. G. L. Verschuur \& K. I. Kellermann (Springer-Verlag, Berlin) 381

Howk, J. C., Ribaudo, J. S., Lehner, N., Prochaska, J. X., \& Chen, H.-W. 2009, MNRAS, 396, 1875

Hyman, S. D., Wijnands, R., Lazio, T. J. W., Pal, S., Starling, R., Kassim, N. E., \& Ray, P. S. 2009, ApJ, 696, 280

Hyman, S. D., Lazio, T. J. W., Kassim, N. E., et al. 2005, Nature, 434, 50

Hyman, S. D., Lazio, T. J. W., Kassim, N. E., \& Bartleson, A. L. 2002, AJ, 123, 1497

Johnston, S. \& Romani, R. W. 2003, ApJ, 590, L95

Keane, E. F., \& Kramer, M. 2008, MNRAS, 391, 2009

Kocsis, B., Haiman, Z., \& Menou, K. 2007, ApJ, 684, 870

Kramer, M., Lyne, A. G., O’Brien, J. T., Jordan, C. A., \& Lorimer, D. R. 2006, Science, 312, 549

Jaeger, T. R., Mutel, R. L., \& Gayley, K. G. 2009, arXiv:0910.5949

Li, X.-D. 2006, ApJ, 646, L139

Linton, E. T., Atkins, R. W., Badran, H. M., et al. 2006, J. Cosmol. Astropart. Phys., 1, 13 
McLaughlin, M. A., Rea, N., Gaensler, B. M., et al. 2007, ApJ, 670, 1307

McLaughlin, M. A., Lyne, A. G., Lorimer, D. R., et al. 2006, Nature, 439, 817

McLaughlin, M. A., \& Cordes, J. M. 2003, ApJ, 596, 982

Millman, P. M., McKinley, D. W. R., \& Burland, M. S. 1948, Nature, 161, 278

Nigl, A., Zarka, P., Kuijpers, J., Falcke, H., Bähren, L., \& Denis, L. 2007, A\&A, 471, 1099

Osten, R. A., \& Bastian, T. S. 2008, ApJ, 674, 1078.

O'Sullivan, J. D., Ekers, R. D., \& Shaver, P. A. 1978, Nature, 276, 590

Phinney, S. \& Taylor, J. H. 1979, Nature, 277, 117

Rees, M. J. 1977, Nature, 266,333

Saltzberg, D., Gorham, P., Walz, D., et al. 2001, Phys. Rev. Lett., 86, 2802

Savage, B. D., Lehner, N., Wakker, B. P., Sembach, K. R., \& Tripp, T. M. 2005, ApJ, 626, 776

Scholten, O., Buitink, S., Bacelar, J., et al. 2009, Phys. Rev. Lett., 103, 1301

Scholten, O., Bacelar, J., Braun, R., de Bruyn, A. G., Falcke, H., Stappers, B., \& Strom, R. G. 2006, Astropart. Phys., 26, 219

Shkolnik, E., Walker, G. A. H., Bohlender, D. A., Gu, P.-G., \& Kürster, M. 2005, ApJ, 622, 1075

Spreeuw, H., Scheers, B., Braun, R., Wijers, R. A. M. J., MillerJones, J. C. A., Stappers, B. W., \& Fender, R. P. 2009, A\&A, 502, 549

Turolla, R., Possenti, A., \& Treves, A. 2005, ApJ, 628, L49

Winglee, R. M., Dulk, G. A., \& Bastian, T. S. 1986, ApJ, 309, L59

Yantis, W. F., Sullivan, W. T., III, \& Erickson, W. C. 1977, Bull. Amer. Astron. Soc., 9, 453

Zarka, P., Queinnec, J., Ryabov, B. P., et al. 1997, in Planetary Radio Emission VI, eds. H. O. Rucker et al. (Austrian Acad., Vienna) 101

Zatsepin, G. T., \& Kuzmin, V. A. 1966, Pis'ma Zh. Eksp. Teor. Fiz., 4, 114 [1966, JETP Lett., 4, 78]

Zhang, B., \& Gil, J. 2005, ApJ, 631, L143

Zhu, W. W., \& Xu, R. X. 2006, MNRAS, 365, L16 